\title{
LE LANGAGE DE LA TRANSCENDENCE ${ }^{1}$
}

En un sens, la philosophie commença en Occident comme transition du mythos au logos, et cette transition fut encouragée par Platon et poussée un peu plus loin par Aristote. Mais pour Platon, le domaine du mythe avait toujours sa place. Lorsqu'il venait à faire l'expérience des limites de la connaissance (gnosis), il faisait appel à "l'histoire vraisemblable," à l'allégorie, au mythe. L'ironie du voyage philosophique de l'Occident, selon Heidegger, est que l'inspiration même qui visa à remplacer le mythos par le logos, non seulement élimina le mythos mais reduisit le logos à une pâle ombre de ce qu'il voulait dire pour la philosophie présocratique. Fondamentalement, le logos est un mot portant sur l'ontos (l'Être), qui peut faire son apparition dans notre Dasein (Être-là), le lieu même où l'Être vient à l'expression. Heidegger nous avait avertis des dangers qu'il y a à abandonner la pensée mythique en faveur de ce qu'il a appelé la "pensée représentationnelle," dont l'origine se trouve chez Platon. La pensée représentationnelle, bien que productrice d'une vision technique du monde, nous laisse désespérés et aliénés quant à notre enracinement dans l'Être. Nous voyons bien plus clairement maintenant, qu' auparavant, que le projet philosophique de Heidegger étair une tentative pour nous ramener à la: compréhension de l'Être qui tul ıejetée par Platon.

Si nous sommes la progéniture philosophique de Platon, ainsi que Heidegger insiste à le dire, nous sommes aussi les héritiers de Kant, dont la critique de l'illusion dialectique ne peut être ignorée. La raison tend inexorablement dans un mouvement d'ascendance vers les idées transcendantales, qui sont les concepts de la raison pure. Ces idées sont produites par la raison dans sa recherche de l'inconditionné, 
de la totalité, mais elles demeurent des idées et on ne peut leur attribuer un objet dans l'expérience. Bien qu'exprimant les intérêts les plus élevés de la raison, ces idées transcendantales--Dieu, le Monde dans sà totalité, et l'Ame--ne peuvent jamais devenir des objets de connaissance puisque, ainsi que Kant lui-même l'a dit, "elles sont transcendantales et dépassent par là même les limites de toute expérience." ${ }^{2}$ Kant n'abandonna jamais son insistence sur le fait qu'il ne peut $y$ avoir de connaissance du transcendant; la psychologie rationnelle, la cosmologie rationnelle, et la théologie rationnelle sont toutes également considérées comme illusoires.

Cependant, il est vrai, ainsi que le remărque Ricoeur dans son article "La Liberté selon l'Expérience," que Kan't ré-introduisit Dieu et l'Ame immortelle de même que la Liberté noumenale dans sa philosophie pratique. Ricoeur ajoute que pour Kant ces idées sont postulées, c'est-à-dire qu'elles sont un Verlangen de manière à ce que la raison pratique puisse se donner un but. ${ }^{3}$ Il convient aussi de dire que ces transcendantes totalités ne sont jamais gratifiées du terme honorifique de "connaissance"; elles sont plutôt des "postulats" que la raison dans son déploiement pratique se donne à elle-même de manière à ce que les "glorieuses idées de moralité" puissent devenir "des ressorts pour l'action." ${ }^{4}$ Bien que Kant ait exclu toute possibilité de connaissance de ce qui transcende nos sens, il a affirmé qu'il acceptait néanmoins "le langage tout à fait légitime d'une foi ferme.".5 Mais il se trouve que dans sa philosophie pratique, le seul langage de la foi est le langage de la moralité. L'âme est simplement la possibilité de la liberté nouménale et Dieu est seulement le guarant d'une vie future durant laquelle le summum bonum peut être realisé, le summum bonum étant le royaume des fins dans lequel la morale et la vertu coïncident et le bonheur donné à ceux qui méritent d'être heureux.

En un certain sens, la philosophie critique de Kant peut être considérée comme le triomphe de la pensée représentationnelle. Les limites des sens constituent les paramètres à la fois de ce que l'on peut légitimement appeler connaissance et de ce qui peut se présenter à notre entendement comme représentation. L'intention de Kant en tout 
cela était d'honorer la philosophie en l'élevant au niveau d'une science; mais ce faisant, il établit involontairement les fondations du positivisme qui prolongea l'attaque kantienne contre la métaphysique dogmatique mais qui par ailleurs dirigea sa propre critique contre le sentiment moral en le réduisant à l'expression d'une préférence subjective, d'une émotion, ou d'un discours persuasif.

En somme, pour Kant, le transcendant ne peut être exprimé car il ne peut être l'objet d'une expérience. La notion d"'expérience" a une signification restreinte enracineée dans les formes a priori de la sensibilité--l'espace et le temps. Il n'y a pas dans le système kantien de place pour le symbolique; le niveau de son discours s'inscrit dans le champ sémantique de la physique et des mathématiques. La physique du temps de Kant, cependant, était le monde sûr et certain de la mécanique newtonienne et de la géométrie euclidienne. Kant n'avait pas anticipé--et son système critique n'est pas à l'abri--les extravagances de l'espace riemanien, la continuité espace/temps de la théorie de la relativité, et l'abandon de l'espace et du temps absolus qui constituaient les points de référence de sûreté de la physique newtonienne.

Une autre lacune chez Kant se trouve dans le manque d'attention au langage de la science elle-même et au rôle de la métaphore même dans la physique newtonienne; parler de "champs," de "forces," d" attractions" et de "répulsions," c'est parler le langage de la métaphore. Mais encore plus menaçant pour le système kantien est le fait que la physique des quanta a produit ses propres antinomies: l'univers est fini et limité dû à la "courbature" de l'espace, et cependant en expansion constante; la lumière présente une dualité onde/particule; l'univers est à la fois continu et discontinu. La seconde antinomie de Kant semble aussi avoir une exemplification dans les expériences en physique de haute énergie, qui montrent que lorsque deux particules élémentaires entrent en collision avec une très haute énergie, elles se brisent mais les particules qui en resultent ne sont pas pour autant plus petites que celles qui ont été fissurées dû à l'effet de l'énergie de la collision. Pour la nouvelle physique, il est à la fois vrai et pas vrài que la matière est infinement divisible. 
Une manière de venir à la rescousse de Kant à ce sujet serait d'insister qu'il avait raison dans la mesure où le monde de l'expérience quotidienne était le monde sûr et certain de la physique newtonienne et de l'espace euclidien. Les sens permettent la connaissance des objets, c'est-à-dire de leur position, vitesse, dimension, etc., et nous ne faisons l'expérience d'aucune discontinuité. Au contraire, la physique des quanta a ouvert la voie et par là même rendu possible un nouveau langage pour rendre compte du comportement des particules subatomiques, et ce que nous voyons là n'est pas tant le rejet de la physique newtonienne ou de l'espace euclidien que l'inauguration d'un nouveau mode de compréhension de la-matière-et-de-l'énergie, et d'une nouvelle manière de parler de ces réalités, qui n'est pas issue de l'intuition sensible, au sens kantien, mais qui est de l'ordre de la supersensibilité, à un tel point que Kant ne pouvait l'imaginer.

Mais même l'homme ordinaire intéressé par les science ne peut s'empêcher d'être frappé par la grande différence qu'il y a entre le langage de la physique newtonienne et celui de la physique des quanta, qui apparaît, lorsque jugé par des critères kantiens, plein d'ambiguité de paradoxe et d'un symbolisme les plus abstraits. Le langage de la nouvelle physique n'est pas l'usage littéral du langage; ainsi que le célèbre physicien Werner Heisenberg l'a noté, "tous les mots ou concepts que nous utilisons pour décrire des objets physiques ordinaires, tels que position, vitesse, couleur, dimension, etc., deviennent indéfinis et problématiques lorsque nous essayons de les utiliser pour parler des particules élémentaires." ${ }^{\text {" }}$ Chaque fois que nous sommes confrontés par les limites de l'expérience, au sens kantien, nous. sommes aussi confrontés par les limites du langage, et tout discours au sujet de ce qui transcende une telle expérience doit être métaphorique à l'extrème. Je voudrais insérer ici une distinction que Ricoeur établit en discutant le sense du "texte"; il introduit la distinction entre le monde de l'expérience commune (Umwelt), et d'autre mondes possibles dans le sense de Welt. C'est précisément les révélations rendues possibles par de nouvelles formes d'expression qui donnent naissance à de nouveaux mondes, ou plutôt transforment mon Umwelt en un Welt, et qui par là même rendent possible de nouvelles manières de s'orienter dans ces 
mondes. ${ }^{7}$ Si Kant a décrit notre Umwelt, la nouvelle physique, quant à elle donne naissance d̀ un nouveau Welt.

Bien qu'une partie de la philosophie critique de Kant indique le triomphe de la pensée représentationnelle, on trouve aussi dans son oeuvre l'accent mis sur le rôle constitutif du sujet pensant, sur la seconde "révolution copernicienne"--soulignée avec raison et presentée come l'alternative au scepticisme de Hume. Il ne restait qu'à Husserl, cependant, construisant sur ces foundations kantiennes, de développer les implications de cette révolution en élargissant le sens du terme "expérience" et en étendant le rôle constitutif du sujet connaissant. Deux sorties possibles du cul-de-sac kantien furent proposées par Husserl. Premièrement, Husserl nous enseigna mieux que ses prédécesseurs l'importance du sujet dans la constitution de son monde. Le Cogito n'est plus désormais le sujet pensant vide de Descartes, passivement recevant des impressions, ainsi que la tradition empirique le dit avec insistence, mais il est actif en donnant sens à son monde. La seconde sortie très étroitment liée à la première est l'éli: issement de ce qu'on entend par "expérience." L'expérience n'est plus désormais limitée aux intuitions sensibles, ainsi que Kant l'aurait voulu; avec Husserl, elle est élargie de manière à inclure tout ce qui est présent à la conscience. Renforcé par l'intuition qu'a eu Brentano de la structure intentionnelle de la conscience, Husserl pouvait légitimer la question de la signification de ce dont on est conscient, tout en mettant entre parenthèses les présuppositions au sujet de la réalité de ce qui est présent à la conscience. Par ailleurs, la mise entre parenthèses de la question ontologique rend possible l'ouverture de nouvelles régions d'expérience qui ont leurs propres ontologies régionales.

Une lacune majeure chez Husserl cependant est le manque d'attention au rôle du langage dans la constitution du monde, et c'est à Ricoeur que nous devons l'exploration, dans les traditions les meilleures de la phénoménologie, de la relation entre le sujet, le language et le monde. Le langage n'enferme pas le monde mais permet d'ouvrir de nouveaux mondes. C'est ce que, je pense, Heidegger disait 
par son retour à l'intuition présocratique selon laquelle le logos est le nom à la fois de l'Être et du Dire. ${ }^{8}$

- Je trouve le travail de Ricoeur particulièrement illuminant en ce qu'il montre comment la fonction métaphorique du langage ouvre sur de nouveaux niveaux du discours et par là même sur de niveaux de signification. Mais il est important de se rappeler que Ricoeur a aussi indiqué, en y mettant l'accent, que bien avant toute expression, il y a une précompréhension qui, lorsqu'amenée à l'expression, se trouve toujours traduite dans le langage du symbole. Il est aussi important de situer le traitement du discours symbolique de Ricoeur par rapport à d'autres approches. D'une part, il y a la tentative pour "démythiser" les symboles en les réduisant à une modalité différente, celle qui se concentre sur l'interprétation existentielle au détriment de la forme symbolique originale. D'autre part, il y a une approche purement descriptive, dite phénoménologique, mais en un sens beaucoup plus général qu'il n'est approprié pour la phénoménologie husserlienne, qui essaie quant à elle, de parvenir par delà les formes symboliques aux catégories fondamentales de la conscience mythique. Si la première approche est une tentative pour penser sans symboles, la seconde, elle, est un effort pour penser par-delà les symboles. Ricoeur insiste avec raison, il me semble, que nous devons penser au moyen du symbole: "Le symbole donne à penser." Pour en être sûr, nous devons "démythologiser" le symbole en nous débarrassant du faux logos tenu pour identique avec sa signification littérale, ou alors, ainsi que Tillich le dit, reconnaître la "cassure" des symboles. Alors seulement, l'enterprise herméneutique devient possible.

Deux questions, il me semble, appellent leurs réponses avant que toute herméneutique puisse être entreprise. Si de nouveaux mondes d'expérience possible s'ouvrent quand le langage est poussé vers de nouveaux niveaux de signification, comment choisir entre différentes formes de discours symbolique? Ou à poser la question différemment, quel est le terminus ad quem de l'entreprise herméneutique? D'autre part, et cette question est liée à la précédente, comment pouvons-nous être sûrs que les symboles renvoient à une dimension transcendante de 
l'expérience humaine? Suivant les suggestions de Ian Ramsey, j'apellerai ces questions lc bème de la préférence et le problème de la référence.

Si nous acceptons le point de vue selon lequel le langage de la transcendance est symbolique, la question n'est pas de savoir si nous utiliserons le discours symbolique mais plutôt quelles sortes de symboles nous utilisons. A nouveau, en suivant les recommendations de Ramsey, je suggérerais trois critères pour notre choix de symboles: fertilité, compréhension, et cohérence.

Premièrement, il y a le critère que Ramsey appelle fertilité, et auquel Whitehead se réfère comme étant ce-qui-est-fructueux. De manière à donner une illustration de ce qu'il veut dire par là, Ramsey constitue, à partir de l'Ancien testament, un vaste catalogue de symboles utilisés pour parler de Dieu, un catalogue frappant au départ pour la simple variété qu'il exhibe: Père, Mère, Mari, Ami, Berger, Fermier, Laitière, Blanchisseur, Bâtisseur, Potier, Pêcheur, Marchand, Médicin, Maître, Ecrivain, Infirmière, Forgeron, Roi, Guerrier, et Juge, et sans aucune prétention d'établir une liste exhaustive. La première chose qu'il convient de noter à propos de cette liste est le nombre de symboles qui n'ont pas duré à l'intérieur du discours théologique. Certains n'étaient pas très fertiles, c'est-à-dire qu'ils n'ont pas donné naissance à d'autres formes de discours. Ils ont eu une courte vie alors que d'autres exhibaient une extraordinaire fertilité donnant naissance à d'autres formes symboliques.

Le critère de compréhension est très étroitment lié à celui de fertilité. Les symbu ne doivent pas seulement exhiber une certaine fertilité mais ils doivent être aussi combinés à d'autres symboles et former un système discursif, et plus ils sont compréhensifs, c'est-à-dire plus leur compréhension est grande plus ils pourront ainsi être incorporés. Ceci est, je suppose, une des raisons pour laquelle Ricoeur trouva le mythe d'Adam supérieur aux trois autres expressions mythiques de l'origine du Mal dans l'analyse qu'il développe dans $L e$ Symbolique du mal. Le mythe d'Adam incorpore des aspects des autres 
expressions mythiques à l'interieur d'un jeu dynamique des éléments symboliques, et par là même exhibe une compréhension qu'on ne trouve pas dans ces dernières.

Le troisième critère, celui de cohérence, renvoie non seulement à la cohérence des symboles avec d'autres symbóles mais implique aussi cohérence avec le monde de l'expérience commune. Parler de Dieu comme Amour devra sérieusement prendre en considération la présence du Mal dans le monde, de même que les symboles de Dieu comme Juge devront être conciliés avec la grấce divine. Les symboles doivent aussi être cohérents avec notre expérience du transcendant, et Ramsey remarque que de nombreuses formes du discours symbolique dans les Ecritures ne communiquent plus rien à l'homme moderne à cause de nouvelles situations de vie qui ne sont plus celles qui ont présidé à leur émergence.

Nous ne devons pas assumer qu'il y a une seule forme de discours symbolique. Nous devons toujours rester ouverts à ce que Ramsey appelle "a multi-model discourse," pour lequel nous ne concluerons jamais que la provision de symboles est épuisée.

Pour conclure avec le problème de la référence, je dirais que cette question est en fait la question de savoir comment nous pouvons être sûrs que c'est du transcendant dont nous parlons. Husserl, il me semble, nous a indiqué le chemin à suivre pour éviter les étroitesses du Kantisme qui élimine toute expérience du transcendant, et la question de la référence est fondamentalement une question de signification. Elle ne peut avoir de réponse qu'avec une compréhension élargie du terme "expérience," dans un retour à la précomprehension même, que Otto appelle le numineux, ou que l'on pourrait appeler, pour utiliser les termes de Jaspers, "le saut du Dasein dans l'Existenz," ou encore, ainsi que Ramsey le dit, "une ouverture cosmique," c'est-à-dire une ouverture qui "révèle quelque chose dont nous sommes conscients de l'existence précisément parce que nous sommes conscients d'y être confrontés." ${ }^{10}$ Quand la référence des symboles est mise en question, c'est peut-être le signe que le symbole ne continue plus à 
révéler; il ne donne plus à penser, et un nouveau symbole doit prendre sa place. Je voudrais cependant permettre au Professeur Ricoeur d'avoir le dernier mot en vous laissant avec une question d'un de ses articles les plus récents: "Pourquoi devrions-nous extraire de nouvelles significations de notre langage, si nous n'avons rien de nouveau à dire, pas de nouveaux mondes à projeter?"11

Ohio University

DAVID STEWART

\section{NOTES}

1. Translated by Nader Chokr.

2. Kant, Critique de la raison pure, B384.

3. Kant, Critique de la raison pure, B841.

4. Kant, Critique de la raison pure, B841.

5. Ibid, B773.

6. Werner Heisenberg, Across the Frontiers (New York: Harper \& Row, 1974), p. 144.

7. Ricoeur, "Metaphor and the Main Problem of Hermeneutics," New Literare History 6(1974-75):106.

8. Martin Heidegger, On the Way to Language (New York: Harper \& Row, 1971, p. 80.

9. Ian Ramsey, "Talking of God: Models, Ancient and Mọdern," Christian Empiricism Grand Rapids: Eerdmans, 1974, p. 127ff. En exprimant ma dette à l'égard de l'analyse de Ramsey, je dois en toute 
honnêteté dire que je m'éloigne en quelque sorte du dévelopement qu'il donne de ces thèmes et que je supprime l'usage qu'il fait de ce qu'il considère comme étant un terme neutre "modèle," au lieu de symbole ou métaphore.

\section{Ibid., p. 131.}

11. Ricoeur, "Metaphor and the Main Problem of Hermeneutics," p. 110. 